\title{
Prothèse complète amovible obturatrice implanto-retenue : présentation d'un cas
}

\section{Implant-retained obturator prothesis in hemimaxillectomy: presentation of a case}

\section{Résumé}

MOTS-CLEFS :
- Implant, maxillectomie,
prothèse obturatrice,
rétention, perte
de substance
KEYWORDS:
- Implant, maxillectomy,
obturator prothesis,
retention, maxillectomy
defect

Les grandes pertes de substance maxillaire sont de prise en charge délicate. De plus en plus, les patients maxillectomisés bénéficient d'implants afin de faciliter l'intégration prothétique. Cependant la chirurgie implantaire est soumise aux contraintes anatomiques et post-thérapeutiques imposant au praticien de réaliser des compromis lors de l'élaboration. Nous présentons ici le cas d'un patient qui a pu bénéficier de la pose d'un implant en supplément de rétention de sa prothèse maxillaire complète avec obturateur.

\author{
Abstract
}

Restoring large maxillary defects to a functional and comfortable state for patients is a difficult task. We observe an increase in the use of implants to improve the prosthesis acceptance, but in the case of patients having undergone an hemimaxillectomy and radiotherapy the feasibility of using implants is subordinated to the state of the residual anatomic supports. Hence prosthetical treatment needs to be adapted to each individual case. We present herein the oral rehabilitation of an hemimaxillectomy patient with an implant-retained obturator prosthesis.

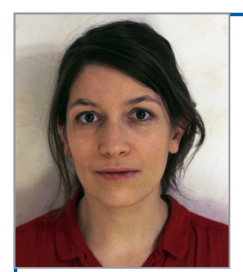

- Alice CROS, Service d'odontologie, CHU de Toulouse, France, UFR odontologie 3 chemin des Maraîchers 31059 Toulouse

Mélanie BOULANGER, Service d'odontologie, CHU de Toulouse, France, UFR odontologie 3 chemin des Maraîchers 31059 Toulouse, Institut-Universitaire du Cancer de Toulouse-Oncopole, Toulouse, France

Bruno COURTOIS, Service d'odontologie, CHU de Toulouse, France, UFR odontologie 3 chemin des Maraîchers 31059 Toulouse

Philippe POMAR, Service d'odontologie, CHU de Toulouse, France, UFR odontologie 3 chemin des Maraîchers 31059 Toulouse

Emmanuelle VIGARIOS, Service d'odontologie, CHU de Toulouse, France, UFR odontologie 3 chemin des Maraîchers 31059 Toulouse, Institut-Universitaire du Cancer de Toulouse-Oncopole, Toulouse, France

\section{INTRODUCTION}

Les pertes de substance maxillaire d'étiologie oncologique sont de prise en charge complexe $[1,18,19,20]$. Elles sont la conséquence d'une chirurgie d'exérèse qui affecte les tissus osseux et muqueux et provoquent une solution de continuité ostéo-muqueuse bucco-nasale, bucco-sinusienne ou bucco-naso-sinusienne. Elles ont pour conséquences des troubles fonctionnels (perte d'étanchéité de la cavité buccale, phonation difficile, mastication perturbée) et psychologiques parfois sévères laissant les patients face à une défiguration 
marquante, les isolant et les stigmatisant définitivement $[2,3]$.

La prothèse obturatrice, quelle que soit la forme de la cavité de la perte de substance, trouve très souvent une rétention et une stabilisation satisfaisantes sur les dents restantes afin d'offrir un résultat esthétique et fonctionnel acceptable chez les patients dentés [26]. Mais ce résultat est plus aléatoire dès lors que les patients sont édentés complets.

Les implants sont de plus en plus proposés dans le traitement des pertes de substance étendues $[4,5,6]$. Ils convient néanmoins de bien sélectionner les patients éligibles qui, dans le contexte de radiothérapie et de chimiothérapie, ont des risques majorés de complications parfois graves même si la radiothérapie ne constitue plus une contre-indication absolue à la mise en place d'implants.

Nous rapportons ici le cas d'un patient, édenté total, ayant subi une hémi-maxillectomie et qui a pu bénéficier de la pose d'un implant permettant d'apporter un complément de rétention à sa prothèse amovible complète obturatrice.

\section{RAPPORT DE CAS}

Le patient est un homme de 63 ans. Il consulte pour instabilité de sa prothèse maxillaire.

Dans ses antécédents médicaux on note un traitement il y a 10 ans par association radio-chimiothérapie
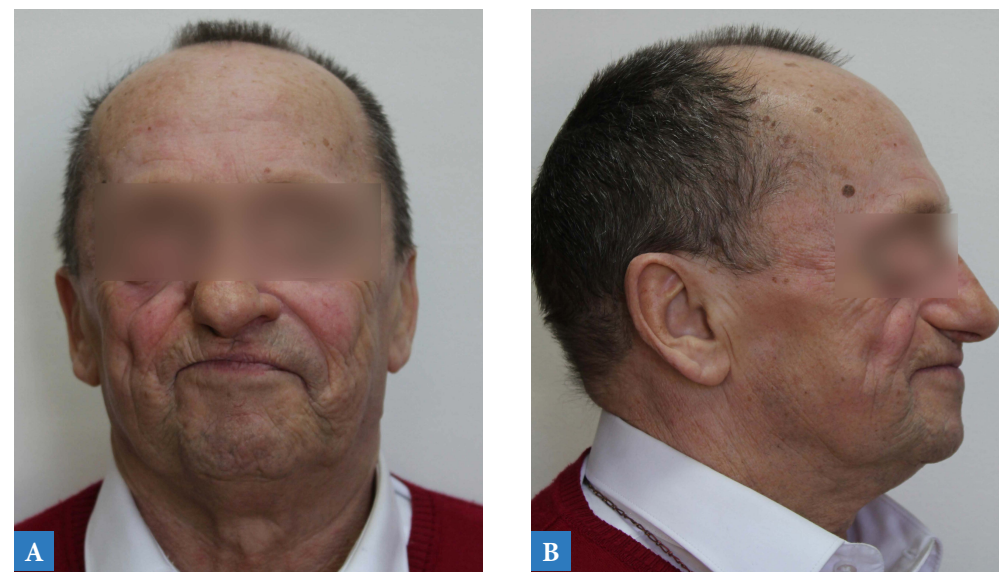

$\triangle$ Fig. 1 : Situation clinique avant traitement :

$A$ et $B$ : vue de face et de profil du patient.

$C$ : vue intrabuccale de la perte de substance.

D : Orthopantomogramme. post chirurgicale suite à un sarcome fusocellulaire du sinus droit.

Dans les antécédents odonto-stomatologiques, on note au maxillaire une perte de substance classe III dans le sens transversal (classification de Pomar et Bentahar, 2008 [7]) : l'édentement est total et la communication bucco-naso-sinusienne se situe sur un hémi-maxillaire emportant tout l'hémi-palais et le pré-maxillaire droit. À la mandibule le patient est en édentement bilatéral terminal (Classe I de Kennedy Applegate), celui-ci est compensé par une prothèse amovible provisoire. La prothèse maxillaire est instable et dysfonctionnelle (alimentation, phonation).

Plusieurs éléments ne sont pas en faveur d'une rétention, stabilisation, sustentation et étanchéité correctes (tétrade prothétique [8]). L'obturateur est volumineux et lourd, favorisant sa désinsertion de la cavité bucco-naso-sinusienne et l'absence d'étanchéité. L'hémi-palais résiduel est plat et les crêtes sont peu marquées. Il est difficile dans l'état actuel de la prothèse d'améliorer la rétention. La prothèse est par ailleurs satisfaisante en terme de rapport inter-arcades et de dimension verticale; elle rétablit correctement les contours du visage et l'esthétique (Fig. 1).

Deux principales options ont été proposées. La première consiste à refaire une prothèse complète avec un obturateur souple en silicone selon la technique de l'empreinte fractionnée de SOULET [7] qui permettrait d'exploiter les quelques reliefs de la cavité bucco-naso-sinusienne comme éléments de rétention
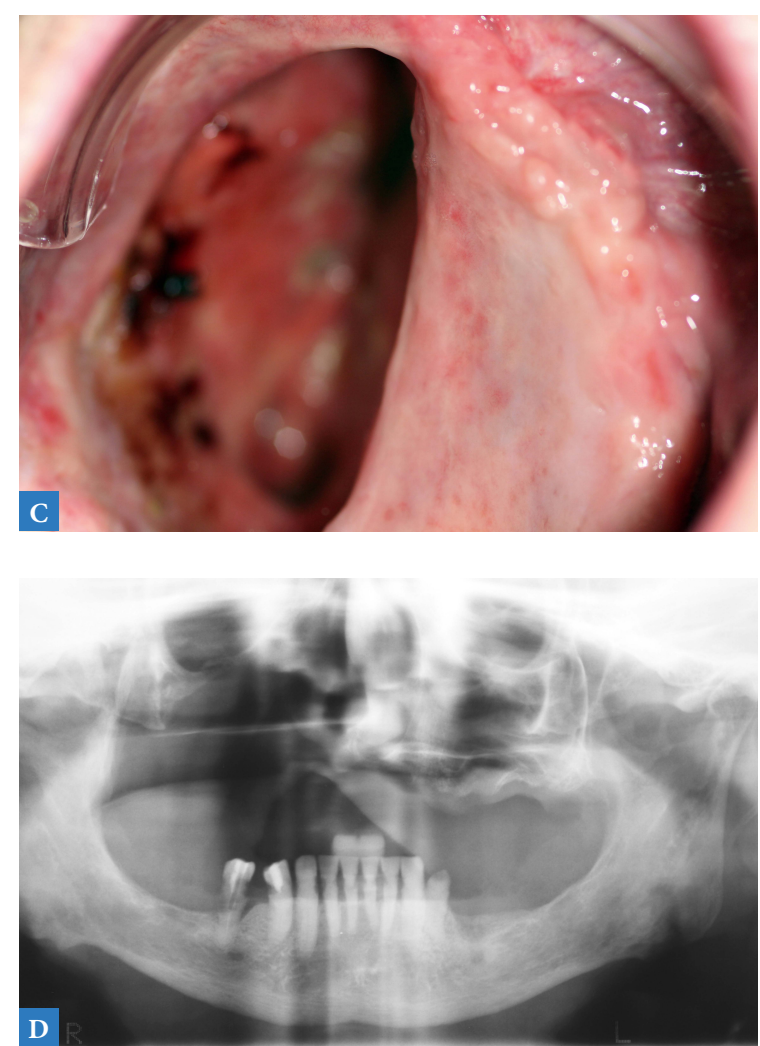


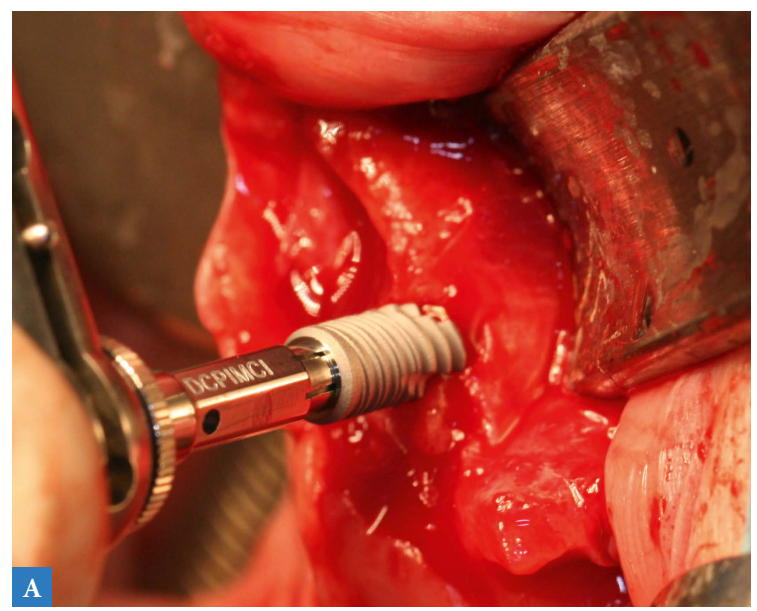

$\triangle$ Fig. 2 : Étapes implantaires.

A : mise en place chirurgicale de l'implant.

$\mathrm{B}$ : cicatrisation gingivale après mise en place de la vis de cicatrisation.

et ainsi de s'opposer aux forces de désinsertion lors des mouvements. La deuxième option, qui a été retenue, à pour objectif de supplémenter la rétention par la pose d'implants. Deux implants sont initialement prévus : un antérieur en position de canine secteur sain et un postérieur en région tubérositaire les deux reliés par une barre ; la liaison avec la prothèse devait être faite par l'intermédiaire d'attachements cavaliers. Cette deuxième option a été validée après discussion avec le patient, les contraintes imposées par l'entretien et la nécessité de réfection de l'obturateur souple n'ont pas retenues son l'intérêt.

La première étape a consisté en une analyse pré-implantaire. Un CBCT a donc été réalisé en utilisant la prothèse maxillaire existante comme guide radiologique avec des repères radio opaques préfigurant la position prothétiquement idéale des deux implants. Il a révélé la faible hauteur osseuse en postérieur pour poser l'implant tubérositaire. La hauteur de crête plus mésialement était également insuffisante, la proximité du plancher sinusien trop proche, pour poser un implant sans réaliser $\checkmark$ Tableau 1:

Étapes

prothétiques. au préalable d'aménagement pré-implantaire type élévation de plancher sinusien. En revanche, en antérieur,

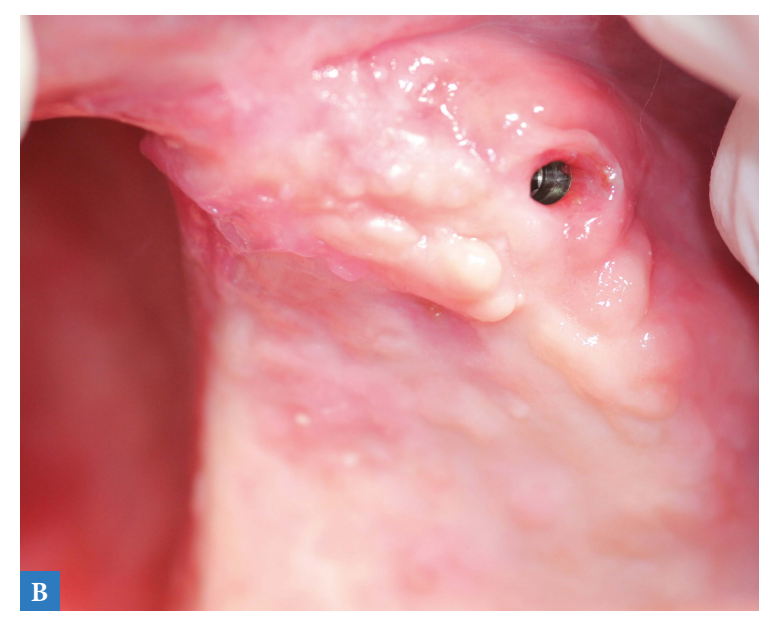

la quantité osseuse résiduelle dans la zone canine était satisfaisante. Les bénéfices et risques liés à la pose d'un seul implant au lieu des deux initialement prévus ont été exposés au patient (surcharge, rotation...) Malgré tout, le patient restait très demandeur d'un moyen de rétention supplémentaire, il a donc été décidé de procéder tout de même à la pose de l'implant antérieur et de concevoir une nouvelle prothèse amovible complète obturatrice (rigide monobloc). Cette réfection a pour objectif d'optimiser la surface d'appui postérieure pour décharger au maximum l'implant en position de repos et lors des mouvements

Après 20 séances d'oxygénothérapie hyperbare, la pose d'un implant a été réalisée sous anesthésie générale (Fig. 2). Un lambeau de pleine épaisseur au niveau crestal puis d'épaisseur partielle a été décollé. Cette approche a permis un accès visuel au site implantaire en limitant l'exposition osseuse. La vascularisation du site était satisfaisante, signe que l'oxygénothérapie a permis d'optimiser sa perfusion. L'implant (4X10 mm, In-Kone ${ }^{\circ}$ universal, Tekka by Global D) a été posé dans un axe le plus parallèle possible à celui de la perte de substance pour ne pas gêner l'insertion de la prothèse. Puis la vis de couverture posée, la muqueuse a été repositionnée, étanchéifiant le site opératoire. L'intervention s'est déroulée sous antibiothérapie (amoxicilline) instaurée jusqu’à cicatrisation muqueuse. Le patient a ensuite suivi 10 séances d'oxygénothérapie hyperbare supplémentaires. Le suivi de la cicatrisation a été réalisé et à six mois post-opératoire, une orthopantomogramme a permis d'objectiver l'ostéointégration de l'implant. La vis de cicatrisation a alors pu être mise en place.

Les étapes de réalisation prothétique ont commencé deux semaines après (Fig. 3). Les étapes de conception d'une prothèse complète conventionnelle supra implantaire et d'une prothèse avec obturateur rigide ont été suivies (Tableau I). 

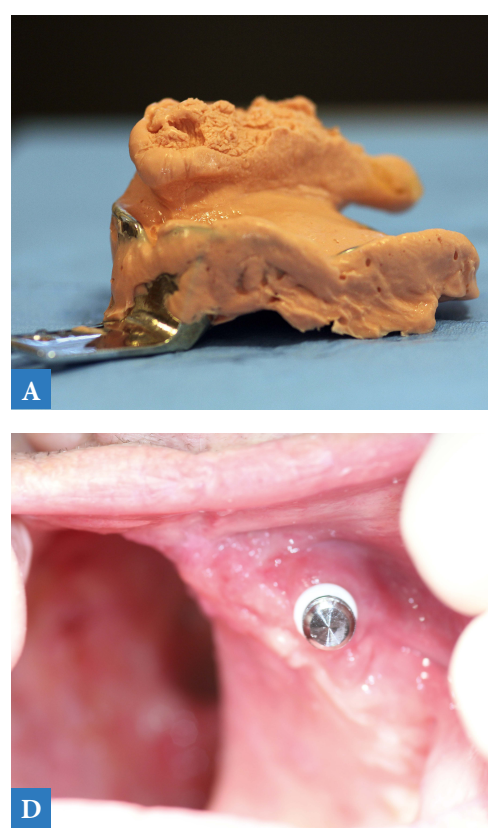

$\triangle$ Fig. 3 : Étapes prothétiques.

A : empreinte primaire.

B : réparation du modèle en plâtre.

C : empreinte secondaire à l'aide d'un PEI.

D : orthopantomogramme.

$\mathrm{E}$ : intrados de la prothèse, obturateur évidé et partie femelle du locator en position.

$\mathrm{F}$ : vue de face du patient avec la nouvelle prothèse.

E
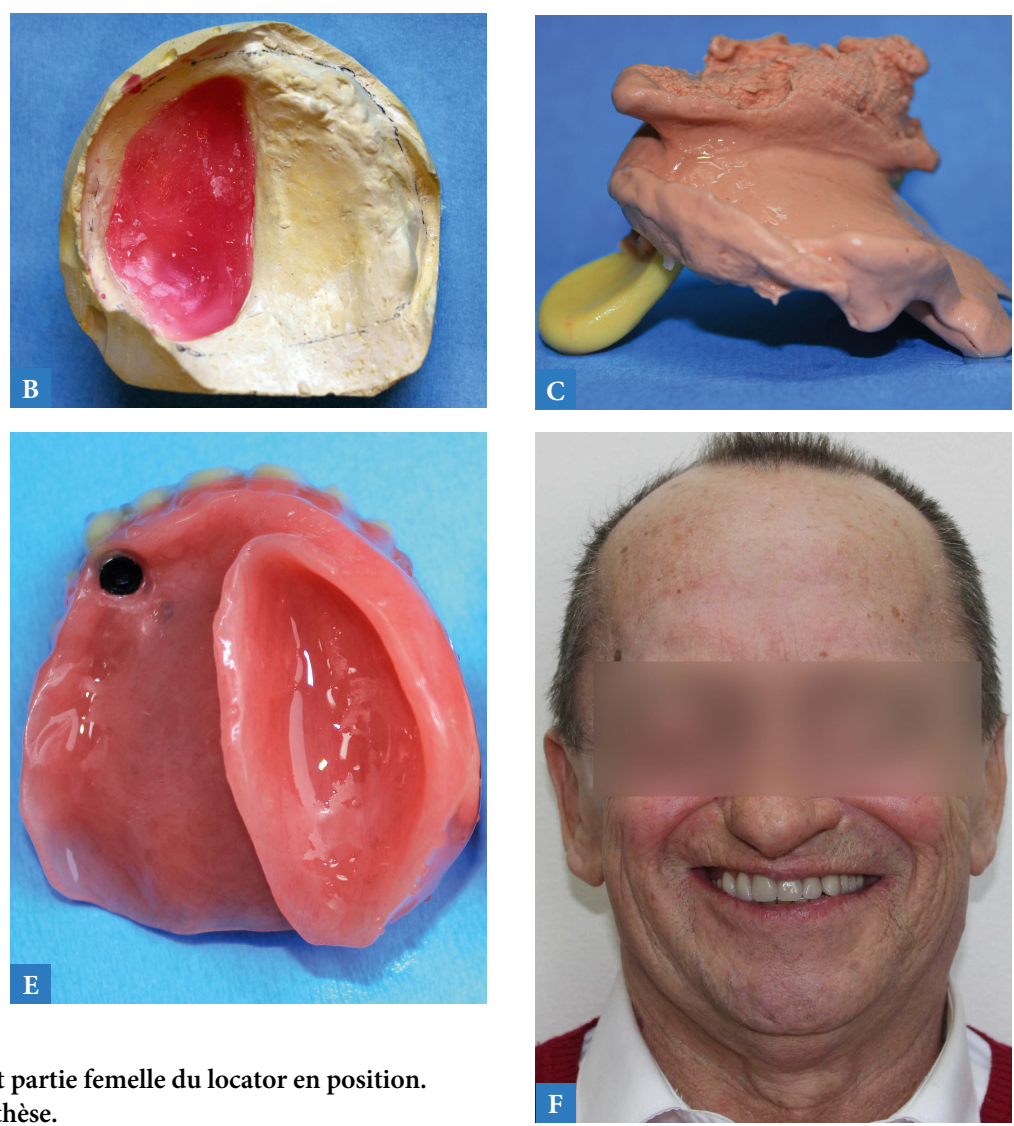

Le type d'attachement Locator ${ }^{\circledast}$ a été retenu pour sa tolérance eu égard à l'axe d'insertion imposé par la cavité d'exérèse. Le positionnement de la partie femelle dans l'intrados prothétique a été effectué en technique directe, sous contrôle occlusal à l'aide de résine polymérisable. La bague de rétention la plus faible a été insérée au départ afin de permettre au patient l'apprentissage de l'insertion et du retrait de sa prothèse avec ce nouvel élément de rétention. L'équilibration de la prothèse amovible complète obturatrice implanto-retenue a été effectuée dans les semaines qui ont suivi l'insertion prothétique.

Le patient porte aujourd'hui sa prothèse depuis 28 mois. La bague du Locator ${ }^{\circledR}$ a été remplacée par celle de rétention supérieure. L'insertion et la désinsertion s'effectuent sans difficulté.

Le patient est suivi régulièrement afin de contrôler les forces exercées sur l'implant.

La prothèse peut être un facteur d'échec à moyen et à long terme de l'intégration de l'implant et de fractures ou dévissages des suprastructures implantaires. On peut observer cliniquement l'apparition de deux spires de l'implant (Fig. 4). Cette dénudation est une complication attendue étant donné le manque de gencive attachée et les contraintes fonctionnelles. Cependant, il n'y a pas de mobilité implantaire, la percussion axiale renvoie un son clair, il n'y a pas de signes pouvant évoquer une péri-implantite. L'implant assure toujours une bonne rétention. Le patient est pleinement satisfait de ce dispositif et rapporte une amélioration considérable de sa qualité de vie orale. Il n'a plus recours à l'utilisation d'adhésifs prothétiques auxquels il était condamné avant.

\section{DISCUSSION}

Les grandes pertes de substance du maxillaire sont de prise en charge délicate. La grande variabilité des formes et tailles des structures résiduelles après l'exérèse d'une tumeur impose une importante adaptabilité thérapeutique tout en respectant un certain nombre de prérequis.

Les conséquences (fonctionnelles, esthétiques...) sont difficilement vécues par les patients qui sont déjà psychologiquement affectés par le diagnostic oncologique et l'incertitude du pronostic. La réhabilitation a pour but de masquer les conséquences de la chirurgie et de permettre au patient de retrouver une certaine forme de banalité de l'apparence [3,23]. Cela permet au patient de recouvrer une vie sociale pour que la guérison psychologique accompagne la guérison physique. Dans ce but il est nécessaire de réaliser des prothèses qui s'intègrent de façon optimale.

Le défaut principal des prothèses obturatrices dans les reconstitutions de classe IIIm chez le patient 

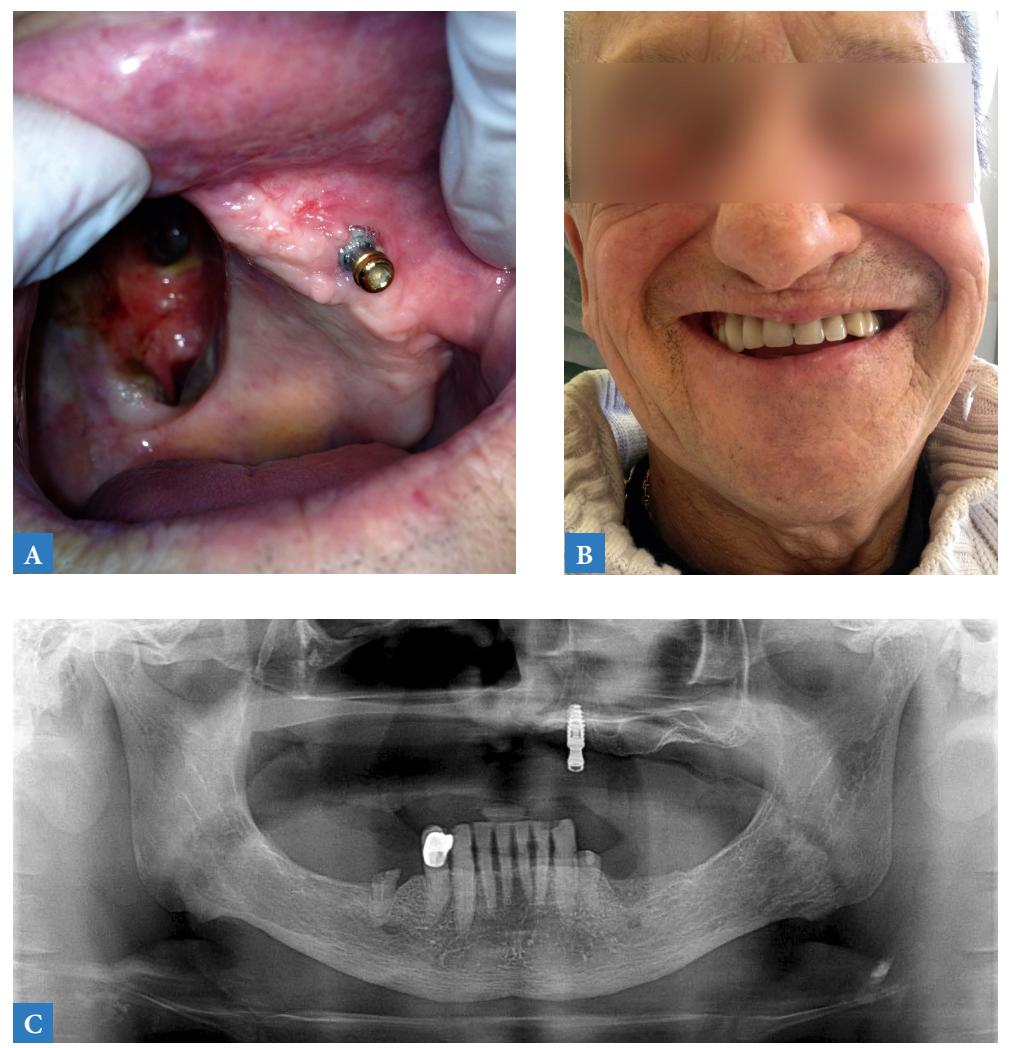

totalement édenté, est le manque de rétention et de stabilité, dépendant de la quantité de tissus résiduels $[20,26]$. Ces éléments entraînent une grande difficulté dans la réalisation des étapes cliniques (exemple : difficulté de stabilisation de la maquette d'occlusion...) et de laboratoire (précision de la mise en articulateur) fiables permettant d'aboutir à une prothèse respectant la tétrade prothétique [8]. À plus ou moins long terme on observe souvent un manque d'étanchéité lors des fonctions orales (mastication, phonation...).

Le plus souvent ces prothèses sont muco-portées. Le choix se fait alors entre un obturateur souple et amovible en silicone ou rigide en résine méthacrylate solidaire de la prothèse. Les obturateurs souples ont de nombreux points négatifs : ils sont difficiles à réaliser car ils nécessitent une technique d'empreinte particulière $[9,10]$ (empreinte fractionnée) et un renouvellement fréquent inhérent au mauvais vieillissement des silicones. La rétention intermédiaire de l'obturateur par attachement magnétique peut être défectueux et nécessiter réparation ou changement. Pour le patient, ils sont plus contraignants car la mise en place se fait en deux temps : d'abord l'obturateur puis la prothèse demandant ainsi une dextérité assez fine. Cependant, ils offrent comme avantages la possibilité d'exploitation des reliefs de la perte de substance comme moyens de rétention et la possibilité de compenser les défauts de parallélisme entre le grand axe de la perte de substance et les structures résiduelles controlatérales. Bien que plus lourds, les obturateurs
Fig. 4 : Réévaluation à 28 mois.

A : implant avec Locator, malgré l'apparition de quelques spires il n'y a pas d'incidence sur la rétention de la prothèse et de périimplantite cliniquement décelable.

B : prothèse en place, l'implant permet toujours d'étanchéifier la cavité bucco-naso-sinusienne et d'apporter un supplément de rétention.

C: orthopantomogramme.

rigides sont plus facilement acceptés par les patients. Ils sont toujours évidés afin d'en réduire le poids. On peut les polir aisément et les réadapter plus facilement. La manipulation et l'entretien de la pièce monobloc sont plus aisés.

De plus en plus, les praticiens ont recours à l'implantologie pour compenser le manque de rétention de ces prothèses. Néanmoins plusieurs précautions sont à prendre chez ce type de patient qui ont souvent reçu une irradiation cervico-faciale dans le cadre de leur traitement oncologique. Tout d'abord, selon les modalités d'irradiation, un risque d'ostéoradionécrose existe. La radiothérapie diminue les capacités de l'os à cicatriser lors d'une agression chirurgicale et peut donc compromettre l'intégration implantaire. La radiothérapie diminue les capacités de l'os à cicatriser correctement autour des implants. En effet, l'os irradié présente des modifications qui sont résumées dans la règle des $3 \mathrm{H}$ : hypocellularité provoquée par la radiothérapie, hypovascularisation entrainant une hypoxie des tissus et une zone à risque d'ostéonécrose, avec une diminution de la capacité à cicatriser. Ce risque n'est pas le même selon le site osseux concerné. Dans une étude sur le squelette maxillo-facial irradié, Granström [12] trouve le plus haut taux d'échec implantaire sur l'os frontal et les sites offrant la meilleure ostéointégration sont l'os temporal et le maxillaire. Cette différence serait le résultat d'une qualité d'os et d'une vascularisation initiale différente ainsi que de la dose cumulée et le temps post irradiation. [13,14]. D'autres facteurs sont à prendre en compte dans ces taux d'échecs tels que l'adjonction d'une chimiothérapie, les morbidités associées, la qualité de l'os implanté, la longueur et le design des implants, le type de chirurgie implantaire, la qualité des tissus mous environnant, l'expérience de l'opérateur [25]. Cependant, les données actuelles ne permettent pas encore de valider et pondérer ces différents facteurs de manière précise.

Face au risque d'ostéoradionécrose, certaines précautions peuvent être prises dans le but de prévenir le développement d'une zone nécrotique post chirurgicale, d'améliorer le pronostic d'ostéointégration et de survie des implants. Parmi les facteurs favorisants proposés on retrouve le sevrage éthylique et tabagique, une hygiène bucco-dentaire correcte, un praticien 
expérimenté et des séances d'oxygénothérapie-hyperbare encadrant l'acte implantaire. L'oxygénothérapie hyperbare $[15,16](\mathrm{OHB})$ consiste à faire respirer le patient de l'oxygène pur à une pression supérieure à celle du niveau de la mer. Selon les recommandations [17], l'OHB favoriserait la prolifération de fibroblastes, et la production de collagène grâce à l'augmentation de la pression en oxygène dans les tissus faiblement perfusés, il y aurait également ralentissement de la perte de la microvascularisation due à la radiothérapie. De nombreuses études ont été réalisées sur l'OHB, sur l'animal d'abord puis chez l'homme montrant que les patients ayant bénéficié des séances d'OHB ont eu une meilleure ostéointégration des implants, que l'angiogénèse est augmentée dès le huitième jour de traitement, qu'elle atteint un palier au vingtième jour et que l'angiogenèse perdure. À trois ans, la vascularisation est égale à $90 \%$ de celle mesurée après les séances. Ces constatations ont permis aux auteurs de proposer un protocole pour l'implantologie en terrain irradié comprenant vingt séances d'OHB en préopératoire et dix séances en postopératoire. L'OHB permettrait donc d'augmenter le taux d'intégration des implants mais d'autres études sont nécessaires pour valider ce protocole.

La position de l'implant, les tissus environnants et les contraintes qui seront appliquées changent également le pronostic, Roumanas et al [22] ont décrit un taux d'ostéointégration supérieur pour les implants posés sur le maxillaire résiduel par rapport aux implants posé dans les berges de la perte de substance, et ce taux était légèrement supérieur pour les implants antérieurs comparé aux implants postérieurs. Cependant les pertes osseuses péri-implantaires à plus long terme étaient supérieures pour les implants antérieurs. L'utilisation des implants est limitée dans les cas de perte de substance maxillaire par la nature du défaut osseux, les cavités sinusiennes et la quantité d'os résiduel disponible. C'est pourquoi le maxillaire antérieur est un emplacement clé. Les auteurs soulignent que bien que les implants antérieurs aient un taux de survie inférieur (après ostéointégration), en lien avec les forces mécaniques endobuccales, ils restent la plupart du temps fonctionnels plusieurs années avant de montrer des signes de mobilité. La perte osseuse péri implantaire est liée en grande partie aux contraintes fonctionnelles prothétiques. Ainsi on peut améliorer le temps de survie en portant une attention très rigoureuse à l'intégration occluso-fonctionnelle de la prothèse.

D'autres sites implantaires sont proposés comme les zygomatiques, les processus ptérygoïdes, du côté de la perte de substance, et la tubérosité maxillaire. Ces positions amènent à de nombreux compromis sur l'accès au nettoyage et l'axe implantaire sera forcément différent de l'axe prothétique. L'absence de gencive attachée conduit également à la réalisation de greffes pour créer un environnement favorable à l'implant. Les implants améliorent la qualité de vie de ces patients en améliorant la rétention des prothèses obturatrices pendant plusieurs années sans manifestation clinique de péri-implantites [27]. C’est dans cette optique que nous avons posé un implant pour aider à la rétention de la prothèse de ce patient. Le fait de ne pouvoir en poser qu'un plutôt que les deux initialement prévus exposait aux risques de blessures par rotation autour de l'axe implantaire, de péri-implantite par surcharge occlusale sur cet unique implant [18] et de complications mécaniques (au niveau de l'implant et des suprastructures). Cependant, la force masticatoire d'un patient édenté total avec une telle perte de substance est largement inférieure à celle d'un patient édenté total sain ce qui réduit les sollicitations implantaires. La prothèse n'aura pas le même impact sur sa survie que dans le cas d'une prothèse conventionnelle. De plus il faut composer avec la spécificité occlusale de ce type de patient [19]. Il ne sera pas possible de retrouver, comme dans le cas d'un patient sain une occlusion généralement équilibrée. En effet, l'absence de base osseuse réduit la sustentation. Même le positionnement d'un implant côté opéré ne pourra pas empêcher les mouvements parasites verticaux au cours de la mastication. Le schéma occlusal bilatéralement équilibré ne sera jamais retrouvé en situation clinique et l'équilibration sera faite selon une occlusion de convenance en statique et en dynamique. Les contraintes occlusales côté opéré sont moins marquées et la dimension verticale d'occlusion est légèrement sous-évaluée (diminution des contraintes sur l'articulation temporo-mandibulaire). L'implant permet surtout de favoriser le confort prothétique auquel aspire le patient. Deux paramètres ont été pris en compte : l'axe de la perte de substance et l'axe de l'implant, les structures résiduelles n'offrant que peu de relief. Le système Locator ${ }^{\varpi}$ utilisé permet de compenser une divergence d'axe de $20^{\circ}$ maximum. $\mathrm{Au}$-delà, il aurait été nécessaire de réaliser un obturateur souple pour supprimer les problèmes d'axe liés à la prothèse puisque seul l'axe de l'implant détermine alors l'insertion. La technique de l'empreinte dissociée $\{29\}$ doit rester une référence pour réaliser de manière précise la liaison entre la suprastructure implantaire et l'intrados prothétique. Cependant, l'unicité de l'implant et les conditions opératoires nous ont fait choisir la méthode directe dans laquelle le contrôle de la fusée de résine en bouche est délicat. Le vieillissement de la résine est par ailleurs rapide et augmente le risque de désolidarisation entre partie femelle et intrados prothétique. 


\section{CONCLUSION}

En raison des différences anatomiques éminemment fréquentes entre les patients maxillectomisés, il n'existe pas de consensus de traitement prothético-chirurgical basé sur des preuves. La réhabilitation des patients est très opérateur-dépendant et fonction des doléances exprimées par les patients. Il faut savoir s'adapter à chaque cas particulier et accepter certains compromis. La situation clinique présentée en a demandé de nombreux notamment pour la partie implantaire. Toutefois, la prothèse remise au patient remplit le cahier des charges de la prothèse obturatrice et permet au patient de retrouver une vie sociale et une qualité de vie satisfaisantes.

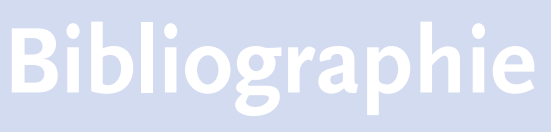

[1] Pomar P, Soulet H. Réhabilitation prothétique après maxillectomie chez l'édenté total : Nouvelle conception de la prothèse obturatrice. Cah. Prothèse 1994, 8

[2] Vigarios E, Fontes-Carrere M, Pomar $\mathrm{P}$, Bach K. Psychologie et relation d'aide en réhabilitation maxillo-faciale. EMC. ; stomatologie, 22-066-B-53,2004

[3] Vigarios E, Destruhaut F, Pomar Ph. Problématique psychosociale en cancérologie cervicofaciale : point de vue de l'odontologiste. EMC médecine buccale, 28-870-M-10, 2010.

[4] Schiegnitz E, Al-Nawas B, Kämmerer P.W, Grötz K.A. Oral rehabilitation with dental implants in irradiated patients: a meta-analysis on implant survival. Clin Oral Invest DOI 10.1007/s00784-013-1134-9

[5] Niimi A, Ueda M, Kaneda T. Maxillary obturator supported by osseointegrated implants placed in irradiated bone : report of cases. J Oral Maxillofac Surg. 1993 Jul, 51 pp.804-9

[6] Barrowman R.A, Wilson P.R, and Wiesenfeld $D$. Oral rehabilitation with dental implants after cancer treatment. Australian Dental Journal, vol. 56, no. 2, pp. 160-165, 2011.

[7] Bentahar O, Pomar Ph, Fusaro S, Benfdil F, Aguenaou A, Abdedine A. Prothèse obturatrice après maxillectomie : bases fondamentales et thérapeutiques. EMC stomatologie 22-066-B-58, 2008.

[8] Destruhaut F, Vigarios E, Toulouse E, Pomar Ph. Divisions palatines non opérées ou séquellaires chez l'adulte : approche fondamentale et thérapeutique en prothèse maxillo-faciale. EMC médecine buccale 28-560P-10, 2011.

[9] Pomar Ph, Soulet H. Empreinte fragmentée : contribution à la réalisation prothétique de l'édenté total après maxillectomie. Actual. Odonto-Stomatol. 1995, $191 ;$ pp 443-7
[10] Parr GR, Gardner K. The evolution of the obturator framework design. J Prosthet Dent, $2003 ; 89: 608-10$.

[11] Roumanas ED, Nishimura SD, Davis BK, Beumer. Clinical evaluation of implants retaining edentulous maxillary obturator prostheses. J Prosthet Dent, 1997 ; 2:184-90.

[12] Granstrom G. Osseointegration in irradiated cancer patients: a analysis with respect to implant failure. J. Oral Maxillofac Surg, 2005, 36, pp.579-585.

[13] Granstrom G. Placement of dental implants in irradiated bone: the case for using hyperbaric oxygen. Journal of Oral and Maxillofacial Surgery, vol. 64, no. 5, pp. 812-818, 2006.

[14] Donoff R.B. Treatment of the irradiated patient with dental implants: the case against hyperbaric oxygen treatment. Journal of Oral and Maxillofacial Surgery, vol. 64, no. 5, pp. 819-822, 2006.

[15] Marx RE, Johnson RP, Kline SN. Prevention of osteoradionecrosis : a randomized prospective clinical trial of hyperbaric oxygen versus penicillin. J Am Dent Assoc 1985 ;111:49-54

[16] Gunepin M., Derache F., Cathelinaud O, Blade JS, Risso JJ, Blatteau JE, Bedrune B, Hugon M, Rivière D. Intérêt de l'utilisation de l'oxygénothérapie hyperbare en prévention de l'échec implantaire chez le patient irradié. Med Buccale Chir Buccale 2012 ;18:143-153

[17] HAS service d'évaluation des actes professionnels oxygénothérapie hyperbare. 01/2007

[18] Nothdurft FP, Propson M, Spitzer WJ, Pospiech PR. Réhabilitation implanto portée d'une perte de substance importante suite à une résection partielle du maxillaire. Rev. Mens Suisse Odontostomatol. Vol 118, 9/2008

[19] Keyf F. Review Obturator prosthese for hemimaxillectomy patients. Journal of Oral Rehabilitation 200128 ; 821-829

[20] Detrait C, Postaire M. Prothèse maxillofaciale chez le patient totalement édenté. Cah Prothèse, 2002 ; 119: p. 17-28
[21] Kornblith AB, Zlotolow IM, Gooen J, Huryn JM, Lerner T, Strong EW, Shah JP, Spiro RH, Holland JC. Quality of life in maxillectomy patients using an obturator prothesis. Head Neck 1996 ; 18:323-334

[22] Nishimura RD, Roumanas E, Beumer J, Moy PK, Shimizu KT, Restoration of irradiated patients using osseointegrated implants : Current perspectives. The journal of Prosthetic Dentistry, 1998 vol 79 n $6,641-647$

[23] Maire F, Kreher Ph, Toussaint B, Dolivet G, Coffinet L. Appareillage après maxillectomie : indispensable facteur d'acceptation et de réinsertion. Rev Stomatol Chir Maxillofac, 2000, $101, \mathrm{n}^{\circ} 1, \mathrm{pp} .36-38$

[24] Giumelli B, Saade K, Le Bars P. Traitement prothétique des pertes de substance acquise des maxillaires en cancérologie. EMC 23-393A- 10,2000

[25] Granström G. Radiotherapy, osseointegration and hyperbaric oxygen therapy. Periodontol 2000 2003, 33, pp.145-162

[26] Margerit J, Pomar Ph, Oussaid M. Rétention en prothèse maxillo-faciale. $E M C$ Médecine Buccale, 28-560-M-10, 2009

[27] Laurito D, Lamazza L, Spink M.J, and de Biase A. Tissue supported dental implant prosthesis (overdenture): the search for the ideal protocol. A literature review. Annali $d i$ Stomatologia, vol. 3, no. 1, pp. 2-10, 2012.

[28] Chan M.F.W.Y, Hayter J.P, Cawood J.I, and Howell R.A. Oral rehabilitation with implant-retained prostheses following ablative surgery and reconstruction with free flaps. International Journal of Oral and Maxillofacial Implants, vol. 12, no. 6, pp. 820-827, 1997.

[29] Mariani P. Prothèses totales à complément de rétention implantaire : l'empreinte dissociée, Synergie Prothétique, pp.229-240, 2001. 